\title{
Further Evidence for the Utility of Electrophysiological Methods for the Detection of Subclinical Stage Retinal and Optic Nerve Involvement in Diabetes
}

\author{
Klára Deák ${ }^{\mathrm{a}} \quad$ Imre Fejes $^{\mathrm{a}}$ Márta Janáky $^{\mathrm{a}}$ Tamás Várkonyi $^{\mathrm{b}} \quad$ György Benedek ${ }^{\mathrm{c}}$ \\ Gábor Braunitzer ${ }^{c}$ \\ Departments of a Ophthalmology, ${ }^{\mathrm{b}}$ Internal Medicine and ${ }^{\mathrm{C} P h y s i o l o g y, ~ F a c u l t y ~ o f ~ M e d i c i n e, ~ U n i v e r s i t y ~ o f ~ S z e g e d, ~}$ \\ Szeged, Hungary
}

\section{Key Words}

Diabetes mellitus · Neuropathy - Visual evoked potentials .

Pattern electroretinography

\begin{abstract}
Objective: To assess the utility of visual electrophysiological methods, visual evoked potentials (VEPs) and pattern electroretinograms (PERGs) were recorded for the detection of subclinical optic nerve and retinal involvement in patients with diabetes mellitus. Subjects and Methods: The data of 63 patients (126 eyes) with no vascular retinopathy or optic neuropathy were retrospectively analyzed. The patients were divided into polyneuropathic/nonpolyneuropathic groups to differentiate between early and late subclinical stages. The recorded parameters were compared with local reference values. Results: 116 eyes (92\%) had VEP and 76 (60\%) had PERG abnormalities. The most frequent alteration was latency delay, but waveform and amplitude irregularities were also observed. The simultaneous use of the two methods allowed us to differentiate abnormal VEPs of purely optic nerve origin from those reflecting retinal involvement. Conclusions: We suggest that regular electrophysiological screening should receive more attention in the ophthalmological care of diabetic patients.

(c) 2015 S. Karger AG, Basel
\end{abstract}

\section{Introduction}

Diabetic retinal neuropathy and optic nerve involvement are frequent complications of diabetes, but they receive little attention in ophthalmological practice since diabetic retinopathy is discussed almost exclusively as vascular retinopathy (i.e. retinal involvement that is evident on fundoscopy) in textbooks and in the literature $[1,2]$. Vascular retinopathy, however, is mostly a late complication [3], only rarely manifest at the time of diagnosis [4]. A patient diagnosed with diabetic retinopathy has approximately a $50 \%$ chance of losing vision in 5 years [3], which in itself calls for efforts to detect retinopathy at the earliest possible stage.

Several methods have been suggested for the detection of subclinical retinopathy, such as fluorophotometry [5] and various electrophysiological methods [3, 6-8]. Evidence suggests that the electrophysiological methods are sensitive and reliable indicators of the retinal and optic nerve involvement in diabetes. However, the literature on this topic is relatively limited, and the significance of electrophysiological methods is clearly underestimated.

In this study, the aim was to provide further evidence for the utility of two electrophysiological methods [vi-

\begin{tabular}{ll}
\hline KARGER & $\begin{array}{l}\text { ○ 2015 S. Karger AG, Basel } \\
1011-7571 / 15 / 0253-0282 \$ 39.50 / 0 \quad \text { Karger }\end{array}$ \\
E-Mail karger@karger.com & $\begin{array}{l}\text { Thisis an Open Access article licensed under the terms of the } \\
\text { Creative Commons Attribution-NonCommercial 3.0 Un- } \\
\text { ported license (CC BY-NC) (www.karger.com/OA-license), } \\
\text { applicable to the online version of the article only. Distribu- } \\
\text { tion permitted for non-commercial purposes only. }\end{array}$
\end{tabular}

Dr. Gábor Braunitzer

Department of Physiology, Faculty of Medicine, University of Szeged Dóm tér 10

HU-6720 Szeged (Hungary)

E-Mail braunitzer.gabor@gmail.com 
Table 1. Alterations of the VEP and PERG values in the polyneuropathy group as compared to the reference values of the laboratory

\begin{tabular}{lcrr}
\hline & Patient & Reference & p value \\
\hline VEP & & & \\
60' N75, ms & $71.42 \pm 10.02$ & $68.93 \pm 5.53$ & 0.955 \\
15' N75, ms & $75.63 \pm 9.37$ & $72.50 \pm 5.07$ & $<0.001$ \\
60' P100, ms & $108.00 \pm 11.76$ & $101.84 \pm 6.28$ & $<0.001$ \\
15' P100, ms & $108.75 \pm 10.22$ & $106.87 \pm 8.06$ & 0.287 \\
60' N135, ms & $153.04 \pm 18.02$ & $142.68 \pm 16.30$ & $<0.001$ \\
15' N135, ms & $149.34 \pm 16.46$ & $140.78 \pm 11.27$ & $<0.001$ \\
60' N135-N75, ms & $81.15 \pm 18.91$ & $73.75 \pm 16.88$ & $<0.001$ \\
15' N135-N75, ms & $74.00 \pm 19.26$ & $68.28 \pm 12.14$ & 0.020 \\
60' N75-P100, $\mu \mathrm{V}$ & $9.13 \pm 5.20$ & $10.25 \pm 6.71$ & 0.344 \\
15' N75-P100, $\mu \mathrm{V}$ & $8.68 \pm 5.00$ & $12.52 \pm 9.32$ & 0.332 \\
60' P100-N135, $\mu \mathrm{V}$ & $10.39 \pm 5.99$ & $11.77 \pm 5.04$ & 0.069 \\
15' P100-N135, $\mu \mathrm{V}$ & $11.37 \pm 6.10$ & $14.04 \pm 5.51$ & $<0.001$ \\
\hline PERG & & & \\
N35, ms & $31.78 \pm 3.57$ & $29.26 \pm 1.75$ & $<0.001$ \\
P50, ms & $54.57 \pm 4.72$ & $50.85 \pm 2.16$ & $<0.001$ \\
N95, ms & $96.04 \pm 9.28$ & $91.48 \pm 5.17$ & $<0.001$ \\
N35-P50, $\mu \mathrm{V}$ & $5.55 \pm 2.46$ & $3.86 \pm 0.99$ & 0.532 \\
P50-N95, $\mu \mathrm{V}$ & $7.22 \pm 3.16$ & $5.33 \pm 1.44$ & $<0.001$ \\
PERG ratio & $1.40 \pm 0.40$ & $1.40 \pm 0.23$ & $<0.001$ \\
RCT 60' (P100-P50), ms & $53.43 \pm 12.82$ & $50.87 \pm 6.96$ & 0.519 \\
RCT 15' (P100-P50), ms & $54.18 \pm 11.11$ & $55.90 \pm 8.56$ & 0.161 \\
\hline
\end{tabular}

Values represent mean \pm SD. $15^{\prime}$ and 60 ' refer to the checksizes used for stimulation. RCT $=$ Retinocortical time.

sual evoked potentials (VEPs) and pattern electroretinograms (PERGs)] in the detection of subclinical retinopathy and optic neuropathy in routine diabetes care.

\section{Subjects and Methods}

In this retrospective study, data of 63 type I diabetes patients with no clinically manifest (vascular) retinopathy were analyzed. The patients were divided into two groups based on the presence or absence of polyneuropathy. Diabetic polyneuropathy was assessed with Neurometer (Neurotron, Inc., Baltimore, Md., USA) and the Ewing test. The assessment was done by an experienced internist who routinely uses these methods (T.V.). Group 1 included 38 patients with polyneuropathy and 1-40 years of diabetes. Group 2 included 25 patients without polyneuropathy and also 1-40 years of diabetes.

Exclusion criteria were any medical conditions that could interfere with the electrophysiological testing, including conditions that affect visual acuity so that it cannot be corrected to 20/20 for the purposes of testing (e.g. cataract). Patients underwent VEP and PERG testing according to the ISCEV standards $[9,10]$.

Black and white checkerboard patterns were used for stimulation. The check size was $60^{\prime}$ and $15^{\prime}$ for VEP and $48^{\prime}$ for PERG recordings. The reversal rate was $0.9 \mathrm{rps}$ for VEP and $2 \mathrm{rps}$ for PERG
Table 2. Alterations of the VEP and PERG values in the polyneuropathy-free group as compared to the reference values of the laboratory

\begin{tabular}{|c|c|c|c|}
\hline & Patient & Reference & $\mathrm{p}$ value \\
\hline \multicolumn{4}{|l|}{$V E P$} \\
\hline 60 N75, ms & $70.42 \pm 10.02$ & $68.93 \pm 5.53$ & 0.728 \\
\hline $15^{\prime} \mathrm{N} 75, \mathrm{~ms}$ & $75.02 \pm 12.97$ & $72.50 \pm 5.07$ & 0.231 \\
\hline $60 ’ \mathrm{P} 100, \mathrm{~ms}$ & $105.06 \pm 11.96$ & $101.84 \pm 6.28$ & 0.206 \\
\hline $15^{\prime} \mathrm{P} 100, \mathrm{~ms}$ & $109.21 \pm 11.22$ & $106.87 \pm 8.06$ & 0.207 \\
\hline $60^{\prime} \mathrm{N} 135, \mathrm{~ms}$ & $146.23 \pm 16.87$ & $142.68 \pm 16.3$ & 0.202 \\
\hline $15^{\prime} \mathrm{N} 135, \mathrm{~ms}$ & $152.29 \pm 20.46$ & $140.78 \pm 11.27$ & $<0.001$ \\
\hline 60' N135-N75, ms & $75.81 \pm 20.49$ & $73.75 \pm 16.88$ & 0.686 \\
\hline 15’ N135-N75, ms & $76.40 \pm 21.19$ & $68.28 \pm 12.14$ & 0.040 \\
\hline 60’ N75-P100, $\mu \mathrm{V}$ & $6.74 \pm 3.65$ & $10.25 \pm 6.71$ & $<0.001$ \\
\hline $15^{\prime}$ N75-P100, $\mu \mathrm{V}$ & $6.80 \pm 3.84$ & $12.52 \pm 9.33$ & $<0.001$ \\
\hline 60’ P100-N135, $\mu \mathrm{V}$ & $7.18 \pm 5.0$ & $11.77 \pm 5.04$ & $<0.001$ \\
\hline $15^{\prime} \mathrm{P} 100-\mathrm{N} 135, \mu \mathrm{V}$ & $8.33 \pm 5.19$ & $14.04 \pm 5.51$ & $<0.001$ \\
\hline \multicolumn{4}{|l|}{ PERG } \\
\hline $\mathrm{N} 35, \mathrm{~ms}$ & $32.77 \pm 3.66$ & $29.26 \pm 1.75$ & $<0.001$ \\
\hline P50, ms & $55.81 \pm 4.65$ & $50.85 \pm 2.16$ & $<0.001$ \\
\hline $\mathrm{N} 95, \mathrm{~ms}$ & $100.1 \pm 8.36$ & $91.48 \pm 5.17$ & $<0.001$ \\
\hline $\mathrm{N} 35-\mathrm{P} 50, \mu \mathrm{V}$ & $3.99 \pm 1.98$ & $3.86 \pm 0.99$ & 0.501 \\
\hline P50-N95, $\mu \mathrm{V}$ & $5.53 \pm 2.54$ & $5.33 \pm 1.44$ & 0.272 \\
\hline PERG ratio & $1.51 \pm 0.54$ & $1.40 \pm 0.23$ & 0.720 \\
\hline RCT 60' (P100-P50), ms & $49.25 \pm 12.56$ & $50.87 \pm 6.96$ & 0.433 \\
\hline RCT 15' (P100-P50), ms & $53.4 \pm 10.99$ & $55.90 \pm 8.56$ & 0.263 \\
\hline
\end{tabular}

Values represent mean \pm SD. 15' and 60' refer to the checksizes used for stimulation. RCT $=$ Retinocortical time.

recordings. Filters were set between 1 and 100 rps for both VEPs and PERGs. The viewing distance was $1 \mathrm{~m}$, and the stimulus display subtended a $12^{\circ}$ by $16^{\circ}$ area. The contrast was $97 \%$. One hundred responses were averaged for VEPs and 200 responses for PERGs. To test trial-to-trial variability, all tests were repeated in the same session after a break of $2 \mathrm{~min}$. Monocular stimulation was applied for VEPs and binocular stimulation for PERGs.

For the VEP recordings, the recording electrode (gold cup) was taped on the $\mathrm{Oz}$ site, the reference electrode on the $\mathrm{Cz}$ site, and the ground electrode on the middle of the forehead (Fz site). For the PERGs, DTL electrodes were used. The reference electrode (gold cup) was placed over either temple, approximately $1 \mathrm{~cm}$ from the ipsilateral orbital rim, and the ground electrode was placed in the Fz site (similarly to VEPs). For the evaluation of VEP alterations the N75, P100 and N135 latencies and the P100 and N135 amplitudes were used. For the evaluation of PERG recordings, the N35, P50 and N95 peak times and the P50 and N95 amplitudes and their ratio (N95/P50) were calculated.

Data from both eyes of each patient were included in the analysis, so as to avoid bias and misinterpretation $[11,12]$. The results were compared with the reference datasets of our laboratory (tables 1,2).

For the comparisons, the Mann-Whitney U test was used, as the criterion of normal distribution was not met. The level of significance was adjusted according to the Šidak correction: $\alpha_{1}=1-$ $(1-\alpha)^{1 / n}$ where $\alpha_{1}$ is the adjusted $p$ level and $\alpha$ is the default $p$
VEP and PERG in the Detection of Subclinical Diabetic Neuropathy 

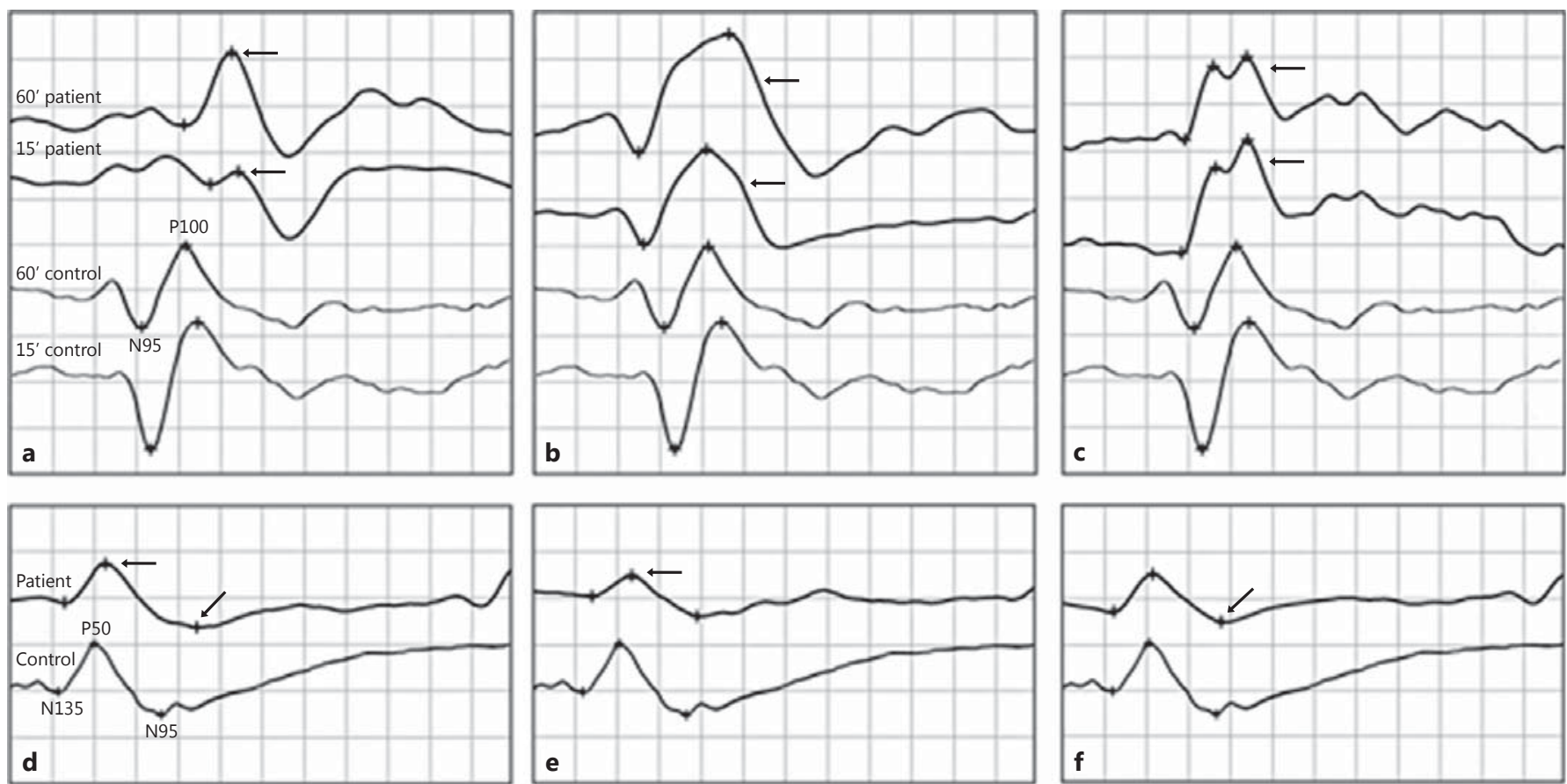

Fig. 1. Characteristic waveform alterations that are seen already when neither polyneuropathy nor the vascular form of retinal involvement is detectable. Recordings from 6 eyes of 6 different patients from the nonpolyneuropathy group. VEP alterations: increased P100 latency in both the 60' and 15' conditions, and a markedly subnormal P100 response in the $15^{\prime}$ condition (a); broad waveforms in both stimulation conditions (b); double P100 peaks

in both stimulation conditions (c). PERG alterations: increased P50 and N95 latency (an elongated response) (d); markedly subnormal P50 response (e); selective N95 attenuation (f). The alterations are indicated by arrows. Calibration: amplitude (abscissa) $5 \mu \mathrm{V} /$ division; time (ordinate) $25 \mathrm{~ms} /$ division. The analytically important peaks and troughs are indicated by crosses. Other conventions are given in boxes $\mathbf{a}$ and $\mathbf{d}$.

level (0.05), and $\mathrm{n}$ is the number of independent comparisons. The adjusted level of significance was $p=0.01$. Statistical analyses were conducted in SPSS 21.0 (IBM, USA).

For stimulation and recording, a Roland Electrophysiological Test Unit was used, with the RETIport 32 software (Roland Consult, Brandenburg an der Havel, Germany). Before the tests, the refractive errors of the eyes were determined and corrected for viewing distance.

\section{Statement of Ethics}

The study was designed according to the Declaration of Helsinki, and it was approved by the Biomedical Research Ethics Committee of the University of Szeged. All patients were informed that their medical data would be used for research purposes, and written informed consent was requested from all the 63 patients. Data were used only upon consent.

\section{Results}

The mean age in the polyneuropathy group was 47.4 years (range: $20-74$ years). The mean diabetes duration in this group was 15.2 years (range: 1-40 years). The

mean age of the polyneuropathy-free group was 49.1 years (range: $21-74$ years). The mean diabetes duration in this group was 14.9 years (range: $1-40$ years).

In the polyneuropathy group, VEP was abnormal in 76 (100\%) eyes, and this was accompanied by PERG alterations in half of the cases, while in the polyneuropathy-free group, VEP was abnormal in 40 eyes accompanied by abnormal PERG in 38 (95\%) eyes. Normal electrophysiological responses were not found in the polyneuropathy group, while in the polyneuropathy-free group normal responses were recorded from 10 (20\%) eyes. Examples of the characteristic alterations are shown in figure 1 . In the polyneuropathy group, the leading alteration was an abnormal delay of P100 which was seen in 62 (82\%) eyes. Doubled P100 peaks and abnormally broad waveforms were also observed but only sporadically (doubled peaks in 6 eyes and broad waveforms in 8 eyes).

PERG findings were abnormal in $38(50 \%)$ eyes. Of the 76 eyes, P50 peak time was delayed in 28 (37\%) eyes, and in 16 eyes subnormal P50 (N35-P50) and N95 (P50-N95) 
peak-to-peak amplitudes were found (21\%). The depression of the N95 amplitude was particularly characteristic (table 1).

In the polyneuropathy-free group, the most frequently observed alteration was also an abnormal P100 delay which was seen in 21 (42\%) eyes. As for the most frequent PERG abnormalities in this group, P50 peak time was delayed in 24 (48\%) eyes, and subnormal P50 and N95 amplitudes were found in 11 (22\%) eyes (table 2).

\section{Discussion}

In this study, the VEP and PERG were sensitive indicators of subclinical retinal and optic nerve involvement in diabetes even at an early stage when the patients were free of neuropathy and the fundus showed no signs of retinal involvement. These methods are also less time-consuming than normal and multifocal ERGs that require dark adaptation and pupil dilation [13]. Equally important, PERG and VEP are best used in combination for the purposes of ophthalmological screening in diabetes. Given the continuity of the optic nerve with the retina, an abnormal VEP recording could indicate either optic neuropathy or retinopathy or both. This differential diagnostic problem would be resolved by the simultaneous use of electroretinography.

As for the specific abnormalities found in this study, especially the peak delays and subnormal responses appear to be characteristic of the studied diabetic patient populations, while less frequent alterations, like double
P100 peaks, definitely require further corroboration. However, it must be taken into consideration that the aim of this study was not to categorize the alterations that can be found early in the course of disease progression, but to investigate if they can be found at all. Our results show that the alterations can be detected, and we suggest that the qualitative details be considered as data to be confirmed or disproven by later studies.

\section{Conclusions}

Our findings showed that PERG and VEP were sensitive tools for the early detection of neural damage, well before the retinal involvement becomes evident on fundoscopy. Therefore, we suggest that regular electrophysiological screening should receive more attention in the ophthalmological care of diabetic patients with the diagnosis of the disease.

\section{Acknowledgments}

Prof. György Benedek was supported by the OTKA Hungary Grant No. K83810. The authors would like to express their gratitude to Prof. Michele G. Shedlin at NYU College of Nursing for her assistance with editing.

\section{Disclosure Statement}

The authors have no conflicts of interest to disclose.

\section{References}

1 Kanski JJ, Bowling B: Clinical Ophthalmology: A Systematic Approach. Oxford, Elsevier Saunders, 2011

2 Lang G, Lang G: Retina; in Lang G (ed): Ophthalmology. A Short Textbook. New York, Thieme, 2000.

3 Adams AJ, Bearse MA Jr: Retinal neuropathy precedes vasculopathy in diabetes: a functionbased opportunity for early treatment intervention? Clin Exp Optom 2012;95:256-265.

4 Al-Zuabi H, Al-Tammar Y, Al-Moataz R, et al: Retinopathy in newly diagnosed type $2 \mathrm{di}$ abetes mellitus. Med Princ Pract 2005;14: 293-296.

5 Cunha-Vaz J, Faria de Abreu JR, Campos AJ: Early breakdown of the blood-retinal barrier in diabetes. Br J Ophthalmol 1975;59:649-656.
6 Ciulla TA, Amador AG, Zinman B: Diabetic retinopathy and diabetic macular edema: pathophysiology, screening, and novel therapies. Diabetes Care 2003;26:2653-2664.

7 Bresnick GH, Palta M: Oscillatory potential amplitudes. Relation to severity of diabetic retinopathy. Arch Ophthalmol 1987;105: 929-933.

-8 Parisi V, Uccioli L, Monticone G, et al: Electrophysiological assessment of visual function in IDDM patients. Electroencephalogr Clin Neurophysiol 1997;104:171-179.

9 Odom JV, Bach M, Brigell M, et al: ISCEV standard for clinical visual evoked potentials (2009 update). Doc Ophthalmol 2010;120:111-119.
0 Bach M, Brigell MG, Hawlina M, et al: ISCEV standard for clinical pattern electroretinography (PERG): 2012 update. Doc Ophthalmol 2013;126:1-7.

11 Fejes I, Kocsis PB, Benedek G, et al: Interocular amplitude and latency differences of pattern ERG and pattern VEP parameters. Optom Vis Sci 2014;91:472-476.

12 Murdoch IE, Morris SS, Cousens SN: People and eyes: statistical approaches in ophthalmology. Br J Ophthalmol 1998;82:971-973.

13 McCulloch DL, Marmor MF, Brigell MG, et al: ISCEV standard for full-field clinical electroretinography (2015 update). Doc Ophthalmol 2015;130:1-12.
VEP and PERG in the Detection of Subclinical Diabetic Neuropathy 